\title{
The Polarised Bent Jet of 3C 43
}

\author{
F. Mantovani ${ }^{1}$, W. Junor ${ }^{2}$, D. J. Saikia ${ }^{3}$ and C. J. Salter ${ }^{4}$ \\ ${ }^{1}$ Istituto di Radioastronomia, Via P. Gobetti, 101, 40129 Bologna, Italy \\ fmantovani@ira.cnr.it \\ 2 Department of Physics and Astronomy, University of New Mexico, Albuquerque, NM 87131, USA \\ bjunor@as.unm.edu \\ ${ }^{3}$ NCRA, TIFR, Post Bag 3, Ganeshkhind, Pune, 411007, India \\ djs@ncra.tifr.res.in \\ ${ }^{4}$ Arecibo Observatory, HC3 Box 53995, Arecibo, Puerto Rico, PR 00612, USA \\ csalter@naic.edu \\ Received 2002 June 26, accepted 2002 December 9
}

\begin{abstract}
Radio observations of linear polarisation provide a useful probe of the physical conditions in the gaseous environments of compact steep spectrum (CSS) sources. Here, we present polarimetric VLBA observations at $8.4 \mathrm{GHz}$ of the CSS quasar 3C 43. The jet in this source curves gently up to about 220 mas from the core, at which point it bends sharply, probably due to a jet-cloud encounter. Regions of polarised emission are detected along the jet, while the core component shows no significant polarisation.
\end{abstract}

Keywords: galaxies: active — galaxies: jets — galaxies: nuclei — quasars: general — quasars: individual (3C 43) — radio continuum: galaxies

\section{Introduction}

Polarimetric observations of compact steep spectrum (CSS) sources provide a useful probe of the physical conditions in the gaseous environments of these young sources. The number of CSS sources for which detailed polarisation information is available is still rather small. To improve this situation, we are conducting a series of observations to image several sources from a list of CSS sources having reasonable degrees of polarised emission and clear signatures of interaction between radio emission and the environment, namely fractional polarisations that decrease with increasing wavelength, and high values of rotation measure $\left(\mathrm{RM}>450 \mathrm{rad} \mathrm{m}^{-2}\right.$ in the source rest frame).

Results for the first two CSS quasars observed in this project, $0548+165$ and $1524-136$, are available in Mantovani et al. (2002). Data analysis of VLBA observations is still in progress for the sources 3C 119, 3C 147, $3 \mathrm{C} 318,3 \mathrm{C} 343$, and $4 \mathrm{C} 24.61$. We present here the images of 3C 43 obtained from VLBA $8.4 \mathrm{GHz}$ full-polarisation observations.

\section{The CSS Quasar 3C 43}

The radio source $3 \mathrm{C} 43$ is identified with a quasar of $m_{\mathrm{v}}=20.0$ at $z=1.46$, and emits a radio power of $P_{1.7 \mathrm{GHz}}=10^{27.8} \mathrm{~W} \mathrm{~Hz}^{-1}$. It has a steep radio spectrum $\alpha_{100}^{1000} \sim 0.7\left(S \propto v^{-\alpha}\right.$; cf. Steppe et al. 1995), and a linear size of $\sim 15 \mathrm{kpc}\left(H_{0}=100 \mathrm{~km} \mathrm{~s}^{-1} \mathrm{Mpc}^{-1} ; q_{0}=0.5\right)$. These characteristics classify $3 \mathrm{C} 43$ as a CSS source. At sub-arcsecond resolution, the radio source has a triple structure with an L-shaped central component. At milliarcsecond resolution, this central component reveals a very asymmetric, bent core-jet morphology (Spencer et al. 1991; Lüdke et al. 1998). A jet-cloud interaction has been suggested (Akujor, Spencer, \& Saikia 1991) as explanation for the sharp bend seen in the jet. VLA observations show that $3 \mathrm{C} 43$ depolarises from $\approx 10 \%$ to $3 \%$ between 15 and $5 \mathrm{GHz}$ (Akujor et al. 1991; van Breugel et al. 1992; Akujor \& Garrington 1995), and the source shows a very high rotation measure of $\mathrm{RM} \sim 1080 \mathrm{rad} \mathrm{m}^{-2}$ in the rest frame of the source (W. Junor et al., in preparation).

\section{Observations and Data Reduction}

$3 \mathrm{C} 43$ has been observed by us with the VLBA ${ }^{1}$, plus one antenna of the VLA. Both hands of circular polarisation were recorded at a frequency of $8405 \mathrm{MHz}$. The source was observed for $5.5 \mathrm{~h}$ in total, together with the calibration sources DA 193, 3C 138 and 3C 286. The data were correlated at the Array Operations Center (Socorro, NM). Data analysis, calibration and imaging were performed using the NRAO AIPS analysis package. Phase gradients in frequency and time were corrected using global fringe-fitting, and polarisation calibration was performed following Cotton (1993). The RCP-LCP delay difference was derived by fringe-fitting a short segment of the crosshand data from a strong calibrator. Strong calibrators were also used to determine the instrumental polarisations of the antennas. The polarisation angle calibration used observations of $3 \mathrm{C} 286$. The data were imaged via the standard iterative self-calibration and imaging processes.

\section{The VLBA Images of $3 \mathrm{C} 43$ at $8.4 \mathrm{GHz}$}

Both full-resolution and low-resolution images of $3 \mathrm{C} 43$ are shown in Figure 1. The rms noise achieved is $0.15 \mathrm{mJy}_{\text {beam }}{ }^{-1}$ in both the low-resolution total intensity

${ }^{1}$ The Very Long Baseline Array and the Very Large Array are facilities of the National Radio Astronomy Observatory, USA 

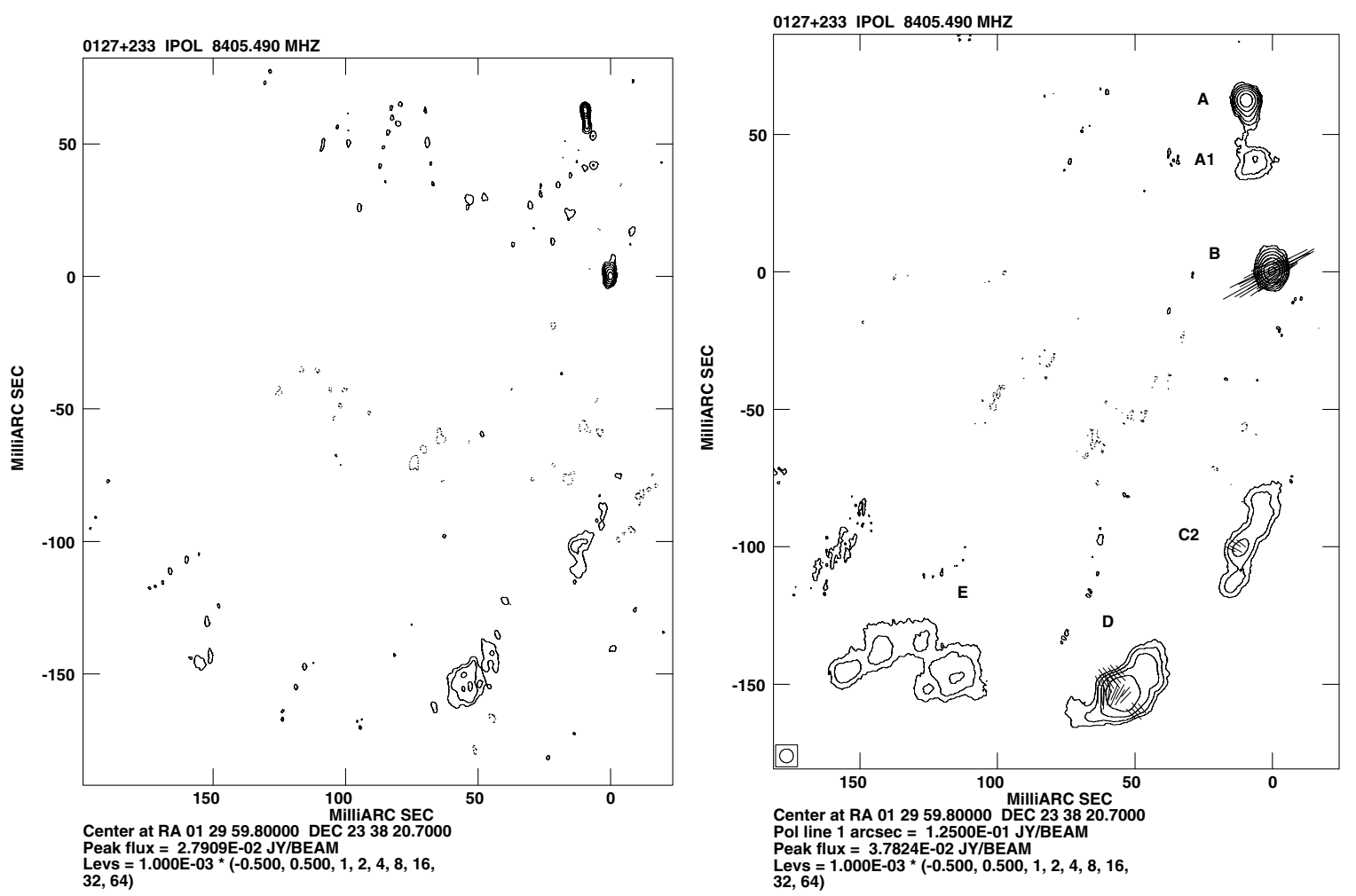

Figure 1 Left: The VLBA image of $3 \mathrm{C} 43$ at full resolution. The beam is $2.4 \times 1.6 \mathrm{mas}^{2}$ at PA $8^{\circ}$. Right: The VLBA image at $8.4 \mathrm{GHz}$ restored with a circular beam of 6 mas. Electric field vectors are superimposed.

Table 1. Source parameters derived from the low-resolution image

\begin{tabular}{lccrrrrr}
\hline Comp & $\begin{array}{c}\text { maj } \\
(\mathrm{mas})\end{array}$ & $\begin{array}{c}\text { min } \\
(\mathrm{mas})\end{array}$ & $\begin{array}{c}\text { PA } \\
(\mathrm{deg})\end{array}$ & $\begin{array}{c}S_{\text {tot }} \\
(\mathrm{mJy})\end{array}$ & $\begin{array}{c}S_{\text {peak }} \\
(\mathrm{mJy})\end{array}$ & $S_{\text {pol }}$ & $\%$ pol \\
\hline A - core & & & & 32.1 & 28.7 & & \\
A - jet & & & & 4.1 & & & \\
A1 & 8.0 & 7.0 & 136 & 5.4 & 2.1 & & \\
B & 3.5 & 1.5 & 177 & 48.4 & 37.8 & 6.0 & 12.4 \\
C2 & & & & 19.3 & 2.8 & 1.1 & 5.7 \\
D & & & & 58.0 & 7.6 & 4.9 & 8.4 \\
E & & & & 34.6 & 2.2 & & \\
\hline
\end{tabular}

The component labels are given following those adopted for the EVN $18 \mathrm{~cm}$ image by Spencer et al. (1991).

and polarised intensity images. The physical parameters derived from the low-resolution image are listed in Table 1.

\section{Results}

The main results coming from these observations can be summarised as follows:

- Component A contains the quasar core. This is concluded as: (a) it shows a clear core-jet structure unresolved at full resolution in the transverse direction; (b) comparing our image with the $18 \mathrm{~cm}$ EVN image of Spencer et al. (1991), it has a 'flat' spectral index, or including the EVN $6 \mathrm{~cm}$ image of C. Fanti et al. (in preparation) it has a convex spectrum which peaks at
$5 \mathrm{GHz}$; (c) the emission is unpolarised down to our detection limit in the low-resolution image.

- The source shows a gently bent, arc-like jet out to knot $\mathrm{D}$, where it displays a sharp, discontinuous bend. This may be due to a jet-cloud encounter in $\mathrm{D}$, as previously suggested.

- Polarised regions of emission are detected along the jet, with polarisation percentages of up to $12 \%$. The polarised flux components seem to lean forwards in regions $\mathrm{B}$ and $\mathrm{D}$, sideways in region $\mathrm{C} 2$. This may indicate bow-shock compression of magnetised plasma.

- In the region containing the jet, the presented VLBA images detect $\sim 50 \%$ of the polarised emission previously measured at $8.4 \mathrm{GHz}$ with the VLA by Akujor \& Garrington (1995). 
- Four regions of polarised emission are detected in component D. Two of these (on the edges of the jet) suggest a similar field direction with the polarisation $\mathrm{PA} \approx 40^{\circ}$. The 'spine' region contains two separate polarised regions, one with $\mathrm{PA}=-30^{\circ}$, and a second, situated more to east, with $\mathrm{PA} \cong 0^{\circ}$. The magnetic field distribution at the edge of the jet could be caused by its interaction with surrounding dense gas clouds while the field in the spine could be due to transverse shocks associated with the flow in the jet. Interaction with the external gas clouds may also be responsible for the deflection of the jet region. A similar finding in the magnetic field distribution along the jet can be seen in the source 1055+018 (Attridge, Roberts, \& Wardle 1999).

- It is interesting to compare the polarisation properties of the CSS sources with the nuclear regions of coredominated quasars. Taylor (2000) noted that in coredominated QSOs the highest RMs are to be found in the inner $20 \mathrm{pc}$ of the nuclear region. In both the quasars reported by Mantovani et al. (2002) large RMs are also found in the jets at distances of a few $100 \mathrm{pc}$ where the radio-emitting material is interacting with dense gas. In $3 \mathrm{C} 43$, the core is unpolarised and the high RM is also likely to be from the bent jet.

\section{References}

Akujor, C. E., \& Garrington, S. T. 1995, A\&AS, 112, 235

Akujor, C. E., Spencer, R. E., \& Saikia, D. J. 1991, A\&A, 249, 337

Attridge, J. M., Roberts, D. H., \& Wardle, F. C. 1999, ApJ, 518, L87

Cotton, W. D. 1993, AJ, 106, 1241

Lüdke, E., Garrington, S. T., Spencer, R. E., Akujor, C. E., Muxlor, T. W. B., Sanghera, H. S., \& Fanti, C. 1998, MNRAS, 299,467

Mantovani, F., Junor, W., Ricci, R., Saikia, D. J., Salter, C., \& Bondi, M. 2002, A\&A, 389, 58

Spencer, R. E., et al. 1991, MNRAS, 250, 225

Steppe, H., Jeyakumar, S., Saikia, D. J., \& Salter, C. J. 1995, A\&AS, 113,409

Taylor, G. B. 2000, ApJ, 533, 95

van Breugel, W. J. M., Fanti, C., Fanti, R., Stanghellini, C., Schilizzi, R. T., \& Spencer, R. E. 1992, A\&A, 256, 56 